\title{
Waiting to Use a Symbol: The Effects of Delay on Children's Use of Models
}

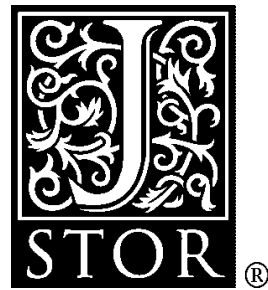

\author{
David Uttal; Jill C. Schreiber; Judy S. DeLoache
}

Child Development, Vol. 66, No. 6 (Dec., 1995), 1875-1889.

Stable URL:

http://links.jstor.org/sici?sici=0009-392028199512\%2966\%3A6\%3C1875\%3AWTUAST\%3E2.0.CO\%3B2-8

Child Development is currently published by Society for Research in Child Development.

Your use of the JSTOR archive indicates your acceptance of JSTOR's Terms and Conditions of Use, available at http://www.jstor.org/about/terms.html. JSTOR's Terms and Conditions of Use provides, in part, that unless you have obtained prior permission, you may not download an entire issue of a journal or multiple copies of articles, and you may use content in the JSTOR archive only for your personal, non-commercial use.

Please contact the publisher regarding any further use of this work. Publisher contact information may be obtained at http://www.jstor.org/journals/srcd.html.

Each copy of any part of a JSTOR transmission must contain the same copyright notice that appears on the screen or printed page of such transmission.

JSTOR is an independent not-for-profit organization dedicated to creating and preserving a digital archive of scholarly journals. For more information regarding JSTOR, please contact support @ jstor.org. 


\title{
Waiting to Use a Symbol: The Effects of Delay on Children's Use of Models
}

\author{
David Uttal \\ Northwestern University
}

Jill C. Schreiber and Judy S. DeLoache

Uniuersity of Illinois at Urbana-Champaign

\begin{abstract}
UtTal, David; Schreiber, Jill C., and DeLoache, Judy S. Waiting to Use a Symbol: The Effects of Delay on Children's Use of Models. Child Development, 1995, 66, 1875-1889. To use a symbol to solve a problem, children must achieve representational insight; they must realize that the symbol stands for its referent. Moreover, they must keep this relation in mind as they attempt to use the symbol. The present studies investigated the achievement and maintenance of representational insight. 3-year-olds were asked to use a scale model of a room to find a toy hidden in the room. In Study la, children first watched as a small toy was hidden in the model. They then waited either $20 \mathrm{sec}, 2 \mathrm{~min}$, or $5 \mathrm{~min}$ before attempting to find a similar, larger toy that was hidden in the corresponding place in the room. All children experienced all delay intervals; three groups experienced the delays in different orders. There was a dramatic effect of delay order. The children who experienced the 20 -sec delay on their first trial generally performed well throughout the 6 trials, but the children who experienced a 5-min delay first almost always failed to find the toy in the room, even on subsequent trials with shorter delays. Additional studies revealed that the negative effects of the initial long delay could be overcome by providing reminders of the model and its relevance (Studies 2 and 3 ) or by giving children prior experience in using the model (Study 4). The results indicate that keeping a symbol-referent relation in mind can be difficult for 3.0-year-old children. This research is discussed in terms of the importance of maintaining representational insight.
\end{abstract}

Symbols are critical to human cognition, culture, and communication. The advent of symbolic capacity, as virtually all theorists of early cognitive development have emphasized, dramatically expands children's intellectual horizons and their potential for learning. Learning to use symbols is thus one of the most important developmental tasks facing young children.

The present studies were motivated by a recent theoretical account of the early development of symbolic understanding (DeLoache, 1990, in preparation; DeLoache \& Marzolf, 1992). In this account, a key prereq- uisite to successful symbol use is representational insight, the basic realization that there is a relation between a symbol and its referent. For example, to use a road map, one must know that it is a representation of a particular area of the world (Liben \& Downs, 1989, 1992). Similarly, to count objects or to do arithmetic, one must realize that numerals represent numerosities (Wynn, 1992).

Several factors are theorized to affect the achievement of representational insight. Instructions are one obvious factor. For example, figuring out what city a street map

Portions of this research were submitted by Jill C. Schreiber in partial fulfillment of the requirements for an M.A. degree at the University of Illinois. In addition, portions of the research were presented at the 1992 annual meeting of the Jean Piaget Society, Montreal. The work was supported by grants from the Spencer Foundation to David Uttal and from the National Institute of Child Health and Human Development (HD-25271) to Judy DeLoache. The work was completed while Uttal was a Postdoctoral Fellow in the Psychology Department at the University of Illinois at Urbana-Champaign (HD-07205). We thank Kathy Anderson and Donald Marzolf for their help with data collection, Renée Baillargeon, Jennifer Banzon, Tonya Bergeson, Debra Janiszewski, Cynthia Fisher, Jeff Lockman, Kevin Miller, Greg Murphy, and two anonymous reviewers for their valuable comments, and the children and staff of the Child Development Laboratory of the University of Illinois. Address correspondence to David Uttal, Department of Psychology, Northwestern University, 2029 Sheridan Rd., Evanston, IL 60208-2710. Address e-mail toduttal@nwu.edu.

[Child Development, 1995, 66, 1875-1889. @ 1995 by the Society for Research in Child Development, Inc. All rights resewed. 0009-3920/95/6606-0005\$01.00] 


\section{Child Development}

represents is much simpler if someone tells us that the large blue area on the right represents Lake Michigan and the heavy black line is Lake Shore Drive. Iconicity, the degree of physical similarity between a symbol and its referent, is a second factor. For example, it is relatively easy to identify bodies of water on maps, because water is typically blue both on maps and in the world (Liben \& Downs, 1989,1992). Past experience with symbols is a third factor. The more exposure one has had to symbolic relations in the past, the more likely one is to figure out a new one. An experienced map reader will more readily realize that something is a map and can more easily match elements on the map to features of the real world (Marzolf \& DeLoache, 1994).

The present research investigated the role of representational insight in very young children's understanding of one type of symbol, scale models. Models have proven to be quite useful for studying the origins and early development of symbolization (DeLoache, 1987). Because scale models are a novel symbol for almost all children, we can investigate representational insight in relation to an unfamiliar symbol. Studying 2.5- and 3.0-year-old children's use of scale models has provided insights into early symbolization that should be applicable to a variety of other symbol systems. ${ }^{1}$

In the scale model task originally reported by DeLoache (1978) and employed in numerous subsequent studies (see DeLoache, 1989, 1991; DeLoache, Kolstad, \& Anderson, 1991; Marzolf \& DeLoache, 1994), young children watch as an attractive miniature toy is hidden somewhere in the scale model of a full-sized room. They are then asked to retrieve a similar but larger toy hidden in the corresponding place in the room itself (Retrieval 1). Finally, the children are asked to return to the model and retrieve the miniature toy (Retrieval 2); this is essentially a memory check. In the previous studies, both 2.5- and 3.0-year-old children have consistently performed very well on Retrieval 2 (over $80 \%$ correct), even when they have performed poorly on Retrieval 1 . Success on Retrieval 2 indicates that any difficulty with Retrieval 1 cannot be attributed to forgetting the location of the original toy.
Representational insight into the modelroom relation supports successful Retrieval 1 performance. To find the toy in the room, children must use their memory representation of the location of the miniature toy in the model to figure out where to search for the larger toy in the room. In other words, they must draw an inference based on the relation between the two spaces: The fact that the miniature toy is located in a particular place in the model means that the larger toy will be hidden in the corresponding place in the room. This inference requires active representations of the model, the room, and the relation between them.

The three factors in DeLoache's account that were mentioned above have been shown to influence whether 2.5- and 3.0year-olds can achieve representational insight for a given model-room relation. Three-year-olds perform very well as long as they receive explicit instructions about the relation (DeLoache, 1989) and there is a high degree of iconicity (physical similarity) between the model and the room (DeLoache et al., 1991). Even 2.5-year-olds can detect a model-room relation if there is an extremely high level of physical similarity and if explicit instructions are given (DeLoache et al., 1991). A third factor that influences both 2.5- and 3.0-year-old children's attainment of representational insight in our model task is their prior experience with similar symbols. Both age groups show significant transfer from easier to harder tasks. For example, 3.0year-olds who first participate - and succeed - in a model task with a high level of similarity between model and room subsequently perform well in the low similarity task that they would otherwise fail (Marzolf \& DeLoache, 1994).

Most previous research with the model task has focused on factors affecting the achievement of representational insight. Successful symbol use requires that one not only achieves but also maintains representational insight. Both terms of the relation must be kept in mind: One's mental representation of the symbol and one's representation of its referent must both be activated to reason from one to the other. Thus, in the model task, children's mental representation of both the model and the room must be ac-

1 Throughout this manuscript, we use the term 2.5 -year-olds to refer to children who are 30-33 months of age. We use the term 3.0-year-olds to refer to children who are 36-40 months of age. These labels are useful for two reasons. First, we want to stress that our age groups comprise a very narrow range of ages. Second, these labels are comparable to those used in reports of previous studies that we have conducted. 
tive if they are to use their knowledge of an event they observe in one space to draw an inference about an unseen event in the other space.

Given how difficult it is for young children to achieve representational insight into a model-room relation, it might also be difficult for them to maintain that relation in working memory. In the research reported here, we examined the effects of a variable that we thought might make it difficult for young children to keep both terms of the model-room relation achieve. Delays were interpolated between the hiding event and the children's first search: After witnessing the experimenter hiding a miniature toy in the model, the children were required to wait varying lengths of time (20 sec, $2 \mathrm{~min}$, or $5 \mathrm{~min}$ ) before searching in the room. We assumed that they would not have trouble keeping the model in mind during the short delay. However, we suspected that longer delays might cause the children to forget about the model and its relevance to the task. If so, they would have no active representation of the model-room relation to serve as a basis for reasoning from one to the other.

It is important to note that the introduction of the delays was the only change from the procedures that have been used in previous studies in which 3.0-year-olds have performed very well. The model was perceptually similar to the room, and we gave children explicit instructions about the model-room relation. Therefore, we assumed that the children would detect the relation between the model, that is, they would achieve representational insight. Thus, the studies presented here focus on the maintenance of, rather than on the initial acquisition of, representational insight.

The first study (Study la) reveals that introducing delays into the model task had surprisingly dramatic effects on 3.0-year-old children's performance. In Study $1 \mathrm{~b}$ we demonstrate that the dramatic delay effect observed in Study la is replicable. In Studies 2,3 , and 4 , we attempt to gain a better understanding of the phenomenon reported in Study 1.

\section{Study 1 a}

\section{Method}

Subjects. - The subjects were $24(12$ male and 12 female) 3.0-year-old children
(36-40 months, $\mathrm{M}=37.7$ months). Eight children (four males and four females) were randomly assigned to each of three groups. In all of the studies reported here, the names of potential subjects were obtained from records of birth announcements, and parents were contacted by telephone. The sample was predominantly white and middle class.

Apparatus and materials. - The study took place in a two-room suite. The larger of the two rooms $(4.80 \times 3.98 \times 2.54 \mathrm{~m})$ was furnished like a living room. It was carpeted and contained a couch, a coffee table, a large armchair, a small wooden dresser, a large floor pillow, a small pillow on the couch, and a built-in bookcase and cabinet unit along one wall. A scale model $(71 \times 65 \times 33 \mathrm{~cm})$ of the larger room was aligned in the same spatial orientation in the second, smaller room. The model was constructed of plywood and was open at the top and one side (the wall opposite the couch and chair) for each access. The model duplicated the main features and furnishings of the room, including carpeting, the built-in wall unit, a window with blinds, and all the items of furniture.

The hidden objects were a stuffed dog $(15 \mathrm{~cm}$ high) and a small plastic dog $(2 \mathrm{~cm}$ high). There was a timer with a beeper to signal the end of the delay, and toys and puzzles to entertain the children during the 2 and 5-min delays.

Procedure.-A parent or preschool teacher accompanied each child to the laboratory. There was a brief play period for the child to become accustomed to the room and the two experimenters. When the child seemed comfortable, the primary experimenter began an extensive orientation. First, she showed the child the larger toy dog and introduced it as "Big Snoopy." She then pointed out and labeled all of the furniture in "Big Snoopy's Room." Next, "Little Snoopy" and his room were presented in the same way. Then the furniture from the model was brought into the larger room, and the experimenter explicitly described and demonstrated the correspondence between all of the items of furniture in the room and in the model. For example, she placed the small couch beside the larger couch and pointed out the similarity between the two. ${ }^{2}$

In a further attempt to communicate the correspondence between the room and the

${ }^{2}$ Although this study was conducted using the model as a symbol for the room, previous research has shown that children perform the same if they first see the toy hidden in the larger room and then search for the corresponding, miniature toy in the model. 


\section{Child Development}

model, the experimenter conducted an imitation placement. She placed the miniature dog on the model table and asked the child to place the large dog in the same place in the larger room. If necessary, assistance was provided. The trials began immediately after the imitation placement.

Each of the six trials involved four parts, beginning with a Hiding Event; the child watched as the experimenter hid the miniature toy in the model, with a different hiding place used for each trial. The six hiding places were: (1)behind the dresser, (2) behind the couch, (3)in the cabinet, (4) under the floor pillow, (5) in the basket, and (6) behind the chair. Half of the children in each group received one of two orders of the six hiding places. The experimenter always called the child's attention to the act of hiding but never referred to the hiding place by name. The child was then told that the experimenter would hide the larger toy in the room, that an assistant would start a timer, and that when the timer rang, the child would go to find the toy in the room.

A Delay Period followed the hiding event. As soon as the experimenter left to hide the second toy, the assistant started the timer to begin one of the three delay intervals: $20 \mathrm{sec}, 2 \mathrm{~min}$, or $5 \mathrm{~min}$. (The $20-\mathrm{sec}$ delay is essentially no delay; i.e., it is the time required for the experimenter to enter the room, hide the toy, and return to the child.) During the 20-sec delays, the assistant talked with the child, and during the longer delays, she played games or solved puzzles with the child. A screen blocked the child's view of the model during the delays.

Retrieval 1 followed the delay. After the timer sounded, the subject was reminded of the correspondence ("Remember Big Snoopy is hiding in the same place as Little Snoopy.") The experimenter then took the child into the room and asked him or her to find the toy. If the child did not find the toy on the first attempt, he or she was encouraged to continue searching other locations. If necessary, the experimenter provided increasingly explicit hints until the child found the toy. The prompts ranged from "Big Snoopy is hiding in the same place as Little Snoopy" (least explicitly) to "I think he's hiding somewhere over there," with a gesture toward the hiding location (most explicit). Thus, the child always retrieved the toy from its hiding location before leaving the room. However, to count as an errorless retrieval (the primary dependent variable), the child's first search had to be correct. After the child retrieved the toy, the experimenter took him or her back to the model.

Retrieval 2 was the fourth and final part of each trial. The child was asked to retrieve the miniature toy that he or she had observed being hidden at the beginning of the trial. Retrieval 2 was thus a standard memory trial. Once again, if the child did not look in the correct place first, he or she was given prompts until the toy was found.

All children experienced all three delays twice, but different groups of children experienced the three delays in different orders. Three orders of delay interval were constructed with these restrictions: (1)Each delay interval occurred once in the first three trials and once in the last three trials; and (2) across subjects, each interval occurred equally often on each of the six trials. The resulting orders are shown in Table 1. Subjects were assigned randomly to one of these three orders, and the resulting groups

TABLE 1

Order in Which the Three Groups in Study la Experienced THE DELAY INTERVALS

\begin{tabular}{cccc}
\hline \hline & \multicolumn{3}{c}{ Group } \\
\cline { 2 - 4 } TRIAL & $\begin{array}{c}\text { Short Delay } \\
\text { First }\end{array}$ & $\begin{array}{c}\text { Medium Delay } \\
\text { First }\end{array}$ & $\begin{array}{c}\text { Long Delay } \\
\text { First }\end{array}$ \\
\hline $1 \ldots \ldots$ & $20 \mathrm{sec}$ & $2 \mathrm{~min}$ & $5 \mathrm{~min}$ \\
$2 \ldots .$. & $2 \mathrm{~min}$ & $5 \mathrm{~min}$ & $20 \mathrm{sec}$ \\
$\mathbf{3} \ldots .$. & $5 \mathrm{~min}$ & $20 \mathrm{sec}$ & $2 \mathrm{~min}$ \\
$4 \ldots$ & $5 \mathrm{~min}$ & $2 \mathrm{~min}$ & $20 \mathrm{sec}$ \\
$5 \ldots .$. & $2 \mathrm{~min}$ & $20 \mathrm{sec}$ & $5 \mathrm{~min}$ \\
$6 \ldots$ & $20 \mathrm{sec}$ & $5 \mathrm{~min}$ & $2 \mathrm{~min}$ \\
\hline
\end{tabular}
first.

NoTE. - The groups were labeled in terms of the delay experienced 
were labeled by the first delay the children experienced; short delay first, medium delay first, long delay first. The three orders were included in the design as a methodological control; however, delay order dramatically affected the children's performance.

\section{Results}

There were two kinds of effects. The most important was a dramatic effect of order on Retrieval 1 performance. The children in the long-delay-first group generally were unable to find the toy in the room, not just on the initial 5-min-delay trial, but on all subsequent trials as well. In contrast, the children in the short-delay-first group usually found the toy in the room. In particular, they performed substantially better than the longdelay-first group after both the $20-\mathrm{sec}$ and 2-min delay trials. The performance of the medium-delay-first group was intermediate but more similar to that of the long-delayfirst group. The second effect of delay interval was on Retrieval 2. There was a straightforward decrease in the children's direct memory for the location of the toy in the model as the length of the delay increased. However, as predicted, this decline was not dramatic; children in all groups generally were able to find the toy in the model.

Retrieval 1 performance.-We focus first on Retrieval 1 performance, which Figure 1 depicts as a function of delay interval and order. The figure illustrates two important results. First, there was a very strong effect of order. Overall, children in the short- delay-first group performed much better $(\mathrm{M}=69 \%)$ than did children in the medium$(\mathrm{M}=38 \%)$ or long-delay-first groups $(\mathrm{M}=$ $19 \%)$. The large group differences were also apparent in the individual data. Six of the eight children in the short-delay-first group found the toy on two-thirds or more of their Retrieval 1 searches. In contrast, only two children in the medium-delay-first group and none of the children in the long-delayfirst group achieved this level of success.

The second main result apparent in Figure 1 is that the effect of delay interval differed across the three orders. Performance varied as a function of delay interval in the short-delay-first order, but not in the other two orders.

These results were revealed in a 3 (delay interval) $\times 3$ (order) ANOVA on the number of errorless Retrieval 1 searches, with delay interval as a within-subjects factor. (All inferential statistics were also calculated with the addition of gender as an independent variable. There were no main effects or interactions involving gender, and hence we deleted this variable in the analyses that we report.) The main effect of order was significant, $\mathrm{F}(2,21)=6.51, p<.01$. Newman-Keuls tests (alpha $=.05$ ) on overall Retrieval 1 performance indicated that the short-delay-first group performed significantly better overall than both of the other two groups, which did not differ from each other. The interaction between delay interval and order was significant, $F(4,42)=$ $2.61, \mathrm{p}<.05$.

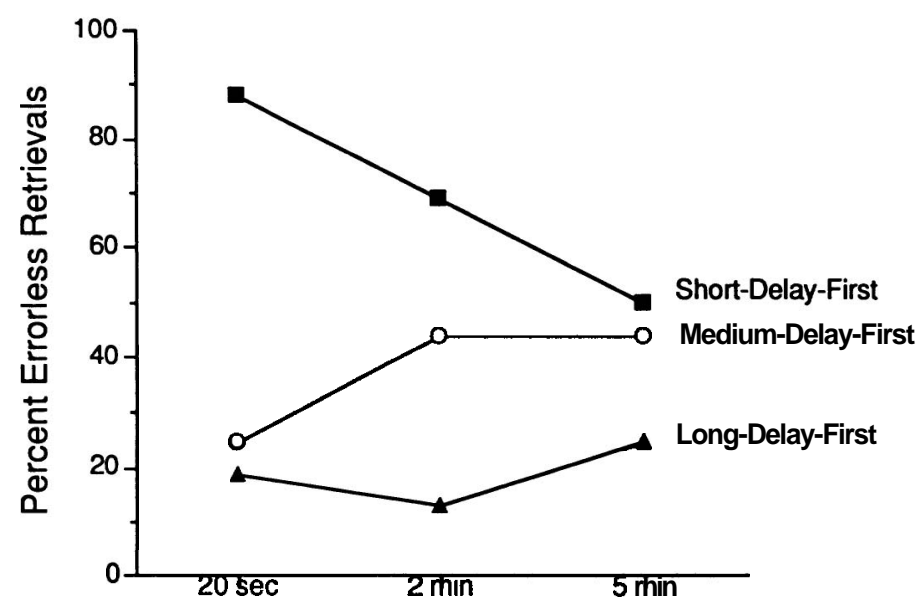

Delay Interval

FIG. 1. - Retrieval 1 performance of the three groups in Study 1 a as a function of delay interval 


\section{Child Development}

Because this interaction was particularly important, two follow-up tests were conducted. Simple effects tests revealed that delay interval affected the performance of the children in the short-delay-first group; they found the toy significantly more often after the 20-sec delays than after the 5-min delays. In contrast, the performance of the medium- and long-delay-first groups was not affected by delay interval; children in these groups performed equally poorly after all delay intervals. Newman-Keuls tests were used to compare the performance of the three groups at each of the delay intervals. The short-delay-first group performed significantly better than both of the other groups after the 20-sec delays and better than the long-delay-first group after the 2min delays. The three groups did not differ after the 5-min delay intervals.

Error analysis. - To gain a clearer understanding of children's performance, we examined their errors and their behavior following these errors. When their first search on a given trial was incorrect, it most often involved going back to a location where they had found the toy on a previous trial. Recall that if they failed to retrieve the toy on their own, the children were prompted as to its location; hence, they retrieved the toy from its hiding place on every trial. Excluding the first trial, on which previous location errors were not possible, $59 \%$ of the children's errors involved searching at a previously correct location. Of these errors, the majority (70\%) were searching at the location where the toy had been hidden on the immediately preceding trial. The prevalence of previouslocation errors is consistent with children's errors in previous model studies (DeLoache et al., 1991), as well as studies of young children's memory for location (e.g., Daehler, Bukatko, Benson, \& Myers, 1976; DeLoache, 1986; DeLoache \& Brown, 1983; Horn \& Myers, 1978; Loughlin \& Daehler, 1973; Perlmutter et al., 1981). Other categories of errors included searching at a new location $(15 \%)$, that is, a location that had never served as a hiding place on a previous trial, and repeatedly searching at a single location (26\%). Spontaneous self-correction (i.e., going immediately, with no prompting, from an incorrect to a correct location) was rare.

Retrieval 2 performance.-We next evaluated the effect of delay interval and order on Retrieval 2 performance, that is, the children's direct memory for where they saw the toy hidden in the model. Overall, children's memory for the location of the toy was excellent ( $82 \%$ errorless retrievals). All but three children (one from each of the three delay order groups) found the toy on twothirds or more of their Retrieval 2 searches. However, the children retrieved the toy less often after the longer delays. The mean level of performance on Retrieval 2 was $90 \%$ after 20 -sec delays, $85 \%$ after 2 -min delays, and $71 \%$ after 5 -min delays. A 3 (order) $\times 3$ (delay interval) ANOVA, with delay interval as a within-subjects factor, was performed on the number of errorless retrievals. The only significant result was the main effect of delay interval, $\mathrm{F}(2,42)=4.26, \mathrm{p}<.05$. Post hoc tests indicated that the children found the toy in the model significantly more often after the 20-sec delays than after the 5-min delays. No other comparisons reached significance. The decline in Retrieval 2 performance, although statistically significant, was not dramatic. The children generally were able to find the toy in the model even after the 5-min delays.

\section{Discussion}

Two different pictures emerge with respect to the effects of delay on children's performance in the model task. Children's direct memory of the location of the hiding event was affected by delay. The Retrieval 2 performance of our subjects declined significantly as the interval between encoding and retrieval increased, just as would be expected from standard memory research from Ebbinghaus (1913) on. Nevertheless, Retrieval 2 performance was still generally very good. All children, on all types of delay trials, usually remembered the location of the toy they saw being hidden in the model.

The picture for Retrieval 1 is quite different, more complicated, and more interesting. Retrieval 1 performance was dramatically affected by delay and delay order. Thus, to characterize Retrieval 1 behavior in this study, we must consider the different delay-order groups separately.

The success of the children in the shortdelay-first group indicates that they clearly gained insight into the model-room relation and, moreover, that during the longer delays they were able to keep active both their representations of the model and of its relation to the room. The only exception was that children in the short-delay-first group performed poorly after the first 5-min delay they encountered (25\%). However, this delay caused only a temporary setback; these children went on to perform very well, even on the second 5-min delay trial (75\%). 
The striking data in this study came from the other two groups, especially the long-delay-first group. It was not surprising that these children did not do well on their first trial with the 5-min delay (38\% correct). It was very surprising that they performed equally poorly or worse on the rest of their trials. Even after the 20-sec delay on trial 2 , their performance was only $13 \%$ correct. For these children, the aftermath of the first 5min delay trial was much more than a temporary setback. Something about the longdelay trial coming first caused these children to fail throughout, reducing their performance to the low level typically shown by 2.5-year-olds in this task (e.g., DeLoache, 1987, 1989).

We think it is unlikely that the problem had to do with gaining an initial awareness of the relation between the model and the room. The present task incorporated two key features that support 3.0-year-olds' detection of the relation between a model and a room: The model was perceptually similar to the room, and we gave children explicit instructions about the relation between them. Thus, all three groups presumably ended the orientation and started the first delay interval equally aware of the correspondence between the two spaces.

We also think it is unlikely that the problem for the long-delay-first group was a simple memory effect. For one thing, Retrieval 1 performance did not vary with delay interval. If straightforward memory effects were involved, then even if children performed poorly after the 5-min delay, we would expect them to do better after the shorter delays. However, the performance of this group was uniformly poor at all intervals. Further evidence against a simple memory explanation is the excellent Retrieval 2 performance of children in all groups, which indicates that the children had little difficulty remembering the location of the toy in the model.

\section{Study 1 b}

The very poor performance of the longdelay-first group of Study la was dramatic and surprising. Therefore, before offering an explanation, we attempted to replicate the finding. We did so in Study $1 b$, modifying the procedure in two ways that should make the results more generalizable. First, we used a different model and room. Second, we included different orders of the three delay intervals. In Study la, each child in the long-delay-first group experienced the delay intervals in the same order (see Table 1).In the present study, all children received a 5min delay on the first trial, but each child experienced the other delay intervals in a different order.

\section{Method}

Subjects. - The subjects (the replication group) were 12 3.0-year-olds (five girls and seven boys) (37-39 months, $\mathrm{M}=37.5$ months).

Apparatus. - Study $1 \mathrm{~b}$ was conducted in a different laboratory than Study la. However, the room, model, and furnishings were similar in the two studies. ${ }^{3}$

Procedure. - The procedures were nearly identical to those of Study la. The only difference involved the order of the delay intervals. Each child received a different one of the 12 possible orders that satisfied the following constraints: (1)The delay on the first trial was always $5 \mathrm{~min}$; (2) each delay interval (20 sec, $2 \mathrm{~min}$, and $5 \mathrm{~min}$ ) had to occur once in trials 1 through 3 and once in trials 4 through 6 . Children were assigned randomly to one of the orders.

\section{Results and Discussion}

The results revealed that the poor performance of the long-delay-first group of Study la is replicable. Although the overall performance of the replication group was somewhat better than that of the long-delayfirst group of Study la, the overall patterns of results was very similar. Most important,

\footnotetext{
${ }^{3}$ The dimensions of the laboratory that was used in Study $1 \mathrm{~b}$ were 21 feet $\times 18$ feet, 4 inches. The room was furnished with two couches, a chair, a coffee table, a small dresser, a small, round table, and a basket. The model was furnished with miniature versions of these items, each of which was perceptually similar (i.e., same color and material) to its referent. The level of similarity between the model and the room was thus somewhat higher in Study $1 \mathrm{~b}$ than in Study la. In Study la, two of the items of furniture in the model were not perceptually similar to their referents. A control group of 3.0-year-olds who participated in the standard model task (DeLoache, 1987) in the laboratory that was used in Study lb performed very well, averaging $88 \%$ correct. This level of performance is slightly better than the level of performance observed in previous model studies.
} 


\section{Child Development}

a one-way ANOVA revealed that the replication group $(\mathrm{M}=38 \%)$ performed significantly worse than the short-delay-first group of Study la $(\mathrm{M}=69 \%)$. In addition, a second one-way ANOVA revealed that the performance of the replication group did not differ significantly from that of the long-delay-first group of Study la. As was true in Study la, performance was not affected by delay interval; average Retrieval 1 performance was $42 \%, 29 \%$, and $42 \%$ after the $20-\mathrm{sec}, 2-\mathrm{min}$, and 5-min delays, respectively. As in Study la, Retrieval 2 performance was good: $71 \%$ correct. Only two of the 12 children (17\%) failed to find the toy on less than two-thirds of the Retrieval 2 searches.

In sum, we obtained the same negative effect of an initial long delay on 3.0-yearolds' performance in the model task in two different studies conducted with different models and rooms and using slightly different procedures. The remainder of this article is devoted to identifying the cause of the initial long delay effect. ${ }^{4}$

\section{Study 2}

In Study 2, we looked further at the possible role of memory in the delay effect. Earlier we noted that it is unlikely that simple memory problems are responsible for the poor Retrieval 1 performance of the longdelay-first group. Retrieval 2 performance was always quite high. However, it is possible that Retrieval 2 does not provide a fully independent measure of children's memory for the location of the toy in the model. Recall that in the previous studies, Retrieval 2 occurred after children had seen the location of the toy in the room on Retrieval 1, either because they had found it themselves, or, if not, because we had given them increasingly explicit hints. consequently, it is possible that the children used their knowledge of the location of the larger toy in the room as a basis for their Retrieval 2 searches in the model. Finding the toy in the room may have reminded them of the correct, corresponding place in the model.

This possibility is remote, because prior pilot work with the model task has shown that children can perform well on Retrieval 2 even when Retrieval 1 is deleted. However, to interpret the delay effect, we must be certain that Retrieval 2 provides a valid, uncontaminated index of children's memory for the original hiding event. We therefore sought to test directly whether children in the long-delay-first condition could remember the location of the miniature toy in the model, independent of finding the larger toy in the room during Retrieval 1, even after the long delay. In Study 2, we reversed the order of Retrieval 2 and Retrieval 1, children searched for the small toy in the model before they searched in the room. Consequently, Retrieval 2 searches could not be contaminated by knowledge of the location of the toy in the room. They therefore provide a valid, unbiased assessment of children's memory for the location of the hiding event.

\section{Method}

Subjects. - The subjects for this study (the reversal group) were eight (four male and four female) 3.0-year-old children (36-40 months, $\mathrm{M}=37.9$ months). Their performance was compared to that of the eight children from the long-delay-first group of Study la (referred to as the standard group). 5

Procedure. - The materials were the same as those used in Study 1. The general procedure was very similar to that described in Study la, with one important difference: Children searched for the toy in the model before searching for the toy in the room. All children participated in the long-delay-first

${ }^{4}$ In the long-delay-first condition, the delay inserted into the first trial necessarily resulted in a substantial gap between when the children received our demonstration of the correspondence between the model and the room and the beginning of the first trial. Consequently, one possible cause of the initial long delay effect is that the children may have forgotten the critically important information that they had learned during the orientation. To determine the locus of the delay effect, we conducted an additional study in which 3.0-year-olds experienced an initial delay, as in Studies la and lb. However, the delay occurred after the initial orientation but before the first hiding event. The children searched for the toy in the room almost immediately after the first hiding event. Performance was much better in this study (65\%)than in Studies la or $\mathrm{lb}$. This result suggests that the delay effect was not attributable to forgetting the information learned during the orientation. The delay effect demonstrated in Study 1 thus stems from the wait between the hiding event and the first search in the room.

5 Throughout the remainder of this article, comparisons will be made to the long-delay-first group of Study la. We chose this group as the standard, rather than the replication group, because they were tested in the same laboratory as children who participated in Studies 2, 3, and 4. 
order of Study la. As in Study la, the delay occurred after the hiding event. At the end of the delay, the children first searched in the model for the miniature toy that they had seen hidden (Retrieval2) and then searched for the larger toy in the room (Retrieval 1).

\section{Results and Discussion}

Because the central question addressed in this study was whether children could remember the location of the toy in the model after the 5-min delay, we consider Retrieval 2 performance first, followed by Retrieval 1 performance.

Retrieval 2 performance. - The performance of the reversal group was very good (85\%)and comparable to that of the standard group (79\%). There was little variation in performance across delays. Thus, the children clearly could remember the location of the toy in the model after any delay, even without seeing the location of the toy in the room. Only one child failed to find the toy in the model on at least two-thirds of the trials. Most of the errors that were made were spontaneously corrected before receiving any prompts, further suggesting that the children remembered where the toy was hidden in the model.

Retrieval 1 performance. - The Retrieval 1 results were quite surprising: the reversal group performed much better than the standard group. As shown in Figure 2, this difference was consistent across all delay intervals. Only the main effect of group was significant in a 2 (group) $\times \mathbf{3}$ (delay interval) ANOVA, $\mathrm{F}(1,14)=10.69, \mathrm{p}<.01$; the reversal group performed significantly better $(60 \%)$ than the standard group $(19 \%)$.

The results of Study 2 provide straightforward evidence that forgetting the location of the toy in the model does not account for the poor performance of the long-delay-first group of Study la. Reversing the order of Retrieval 1 and Retrieval 2 had no impact on Retrieval 2 performance.

The reversal did, however, affect Retrieval 1 performance. Why did finding the toy in the model help children find the toy in the room? Our hypothesis is that returning to the model may have reminded the children of the model and its relevance to the task. To use the model effectively, children must have active representations of the model and the room. In Studies $1 \mathrm{a}$ and $1 \mathrm{~b}$, the initial 5-min delay may have caused the children to forget about the model. Returning to the model after the delay and searching for the toy hidden there reminded them of the model, thereby activating their representation of both terms of the relation between the model and the room.

If this hypothesis is correct, then it might be possible to ameliorate the negative effects of the initial 5-min delay with a more subtle reminder of the existence of the model. Study 3 tested this hypothesis by asking whether simply looking at the model, without searching or seeing the miniature

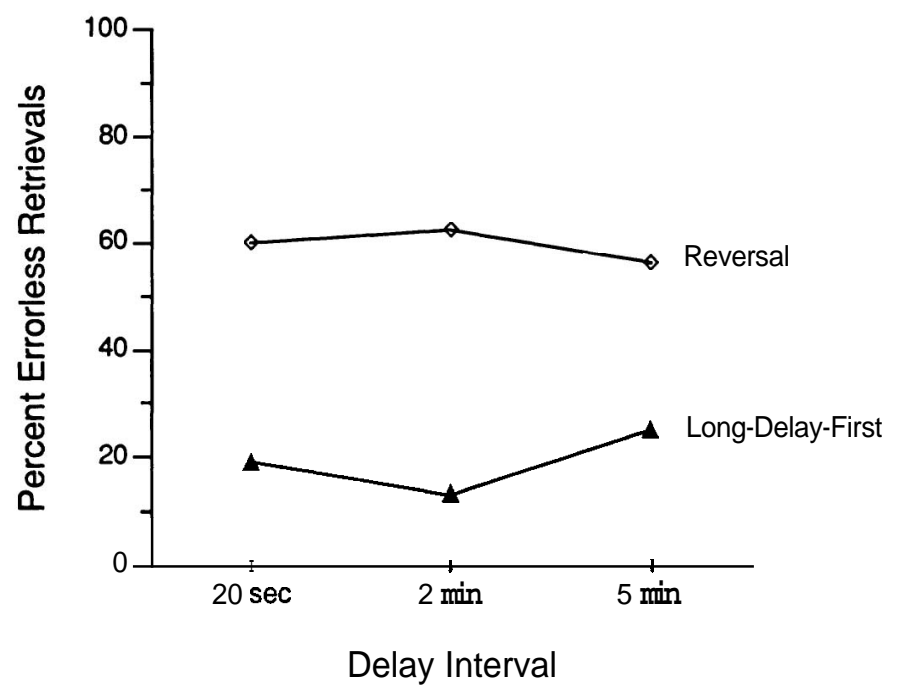

FIG. 2.- Retrieval 1 performance of the Reversal group in Study 2. The performance of the Longdelay-first group of Study la is included for comparison. 


\section{Child Development}

toy, would improve the Retrieval 1 performance of children in the long-delay-first condition. One reason to expect such a minimal intervention to be effective is that in analogical reasoning, even subtle reminders of the relevance of previous problems (e.g., telling subjects that previous problems "might be helpful") can substantially improve performance (Crisafi \& Brown, 1986; Gentner \& Rattermann, 1991; Glick \& Holyoak, 1983; Holyoak, Junn, \& Billman, 1984).

\section{Study 3}

\section{Method}

Subjects. - The subjects in this study, the reminder group, were eight (four male and four female) 3.0-year-old children (36-39.5 months, $\mathrm{M}=37.8$ months).

Procedure. - The materials were the same as those used in the previous studies. The general procedure was similar to the previous studies. Children were tested in the long-delay-first condition of Study la. Retrieval 1 occurred before Retrieval 2, the typical order for model tasks (i.e., they searched for the large toy before they searched for the miniature toy that they had seen hidden). As before, during the delay the model was not visible to the child. The important manipulation in this study was that the subjects were given a visual reminder of the model at the end of the delay. On every trial, before searching for the toy in the room, they were shown the model while they were given the standard verbal reminder ("Remember Big Snoopy is hiding in the same place as Little Snoopy"). The children were not allowed to see the miniature toy in its hiding place or to retrieve it.

\section{Results and Discussion}

The results of this study suggest that this minimal reminder did improve the Retrieval 1 performance, in that half of the children were very successful. The average Retrieval 1 performance was $52 \%$, but the scores were bimodal. Four children found the toy 5 or 6 times (out of a possible 6), and four children found it 0 or 1 times. In contrast, none of the children in the longdelay-first group in Study la found the toy 5 or 6 times. A Fisher's exact test revealed that the performance of these two groups differed significantly, $\mathrm{p}<.05$. (The bimodal nature of the results makes only a nonparametric test appropriate.) As usual, the most frequent Retrieval 1 error in Study 3 was looking in a hiding place at which the toy had been located previously ( $80 \%$ of all errors).
Retrieval 2 performance was very good-96\% errorless retrievals. Seven children out of eight had perfect scores. Thus, even the four children who performed poorly on Retrieval 1 did very well on Retrieval 2.

Study 3 shows that simply seeing the model before searching in the room was sufficient to assist some of the children to overcome the negative effect of the initial long delay. These results suggest that 3.0-yearolds have difficulty maintaining representational insight; they readily forget the relevance of the model for finding the toy.

This explanation suggests that any manipulation that makes the model more salient and memorable in the first place might help to prevent the negative effects of the initial delay. In Study 4, we attempted to inoculate children against the negative effects of an initial long delay by providing them with prior experience using the model successfully. Our assumption was that successful use of the model would help children keep active their representation of the model despite experiencing an initial 5-min delay.

The results of a recent series of transfer studies are consistent with the prediction that prior experience should help children withstand the negative effects of the initial long delay. Experience with a relatively easy model task in which they were successful helped 3.0-year-olds perform well in a more difficult one that they would otherwise have failed (Marzolf \& DeLoache, 1994). The transfer results led us to hypothesize that prior success in the standard model task would enable 3.0-year-olds to cope with the delays that were so problematic to the children in the long-delay-first condition in Study la.

\section{Study 4}

In Study 4, all children participated on 2 days. On the first day, they experienced a model task with no delays, and we expected that they would perform very well, indicating that they had gained insight into the relation between the model and the room. On the second day, all the children participated in the long-delay-first condition of Study la. We hypothesized that prior experience using the model-room relation would diminish the effect of the initial long delay. Specifically, we predicted that successful Day-1 


\section{Uttal, Schreiber, and DeLoache}

performance would result in Day-2 performance that was substantially better than that of the long-delay-first group from Study la.

\section{Method}

Subjects. - The subjects for this study (the prior experience group) were 12 (five male and seven female) 3.0-year-old children (36-49 months, $\mathrm{M}=37.6$ months).

Procedure. - The materials were the same as those used in the previous studies. All subjects were tested on two different days. No more than one day separated the two testings. On Day 1, children participated in six trials of the standard model task with a 20 -sec delay between observing the miniature toy being hidden in the model and searching for the larger toy in the room. On Day 2, they experienced six trials in the same order as the long-delay-first condition of Study la. The procedures used on the two days were identical, except for the different delay intervals.

\section{Results}

The results provide strong evidence that detecting and using the relation between the model and the room on Day 1 enabled the prior experience group to overcome the negative effects of the 5-min initial delay on Day 2. Children who were successful on Day 1 also did well on Day 2, despite the 5-min initial delay.

As expected, overall Retrieval 1 performance on Day 1 was reasonably good $(64 \%)$, although it was somewhat bimodal. Eight of the 12 children were quite successful, searching correctly on two-thirds or more of their six trials. Three children were markedly unsuccessful, finding the toy on only one trial. The remaining child searched correctly on three of the six trials. ${ }^{6}$

More important, the prior experience group in the study also performed well on Day $2(65 \%)$, much better than the longdelay-first group of Study la (19\%) (see Fig. 3 ). A 2 (study) $\times 3$ (delay interval) ANOVA revealed a main effect of study, $F(1,18)=$ $14.09, \mathrm{p}<.001$. Neither the main effect of delay interval nor the interaction was significant; as shown in Figure 3, the children who had prior experience performed well after all delays, and the long-delay-first group performed poorly after all delays.
In addition to the group analyses, it is important to examine individual data to see how prior experience with the model-room relation affected Day-2 performance. One specific prediction is that any child who failed in the no-delay (20-sec) task on Day 1 should be similarly unsuccessful in the longdelay-first condition on Day 2. The data were consistent with this prediction. None of the four children who had Retrieval 1 scores of less than $67 \%$ on Day 1 was successful on Day 2; their average performance on Day 2 was $25 \%$ - the same as their average score on Day 1. Thus, children who did not exploit the easier, no-delay task on the first day apparently remained unaware of the relevance of the model-room relation on the second day.

In contrast, all eight of the children who were successful (i.e., who had $67 \%$ or better errorless retrievals) on Day 1 were similarly successful on Day 2; their average Retrieval 1 performance was $85 \%$. Thus, the children's individual performance was highly consistent with the group data, and both strongly supported the hypothesis that successful Day-1 performance would lead to improved Day-2 performance.

Finally, direct memory for the location of the toy in the model (Retrieval 2) was good, as it was in the previous studies - $86 \%$ on both days. Only one of the 12 children failed to find the toy on fewer than twothirds of the Retrieval 2 searches.

\section{Discussion}

The results of Study 4 indicate that detecting and using the relation between the model and the room on Day 1 inoculated children against the initial long delay. On Day 1 , most of the children realized that the model was related to the room and that information they gained from the model should guide their searching in the room. Therefore, on Day 2, the 5-min initial delay had no effect; the children in Study 4 performed as well as the short-delay-first group of Study la. In addition, successful experience on Day 1 eliminated the effect of delay interval on Retrieval 1; performance was just as good after 5-min delays as after 20 -sec delays. The results are consistent with previous research on transfer in young children's use of symbols (e.g., DeLoache, 1991; Marzolf \& De-

6 The overall level of Retrieval 1 performance was lower than in previous studies with the standard model task. However, note that children in the present study could not see the model during the 20-sec delay on each trial on Day 1 . In previous studies, the model was visible while the experimenter was hiding the toy in the room. 


\section{Child Development}

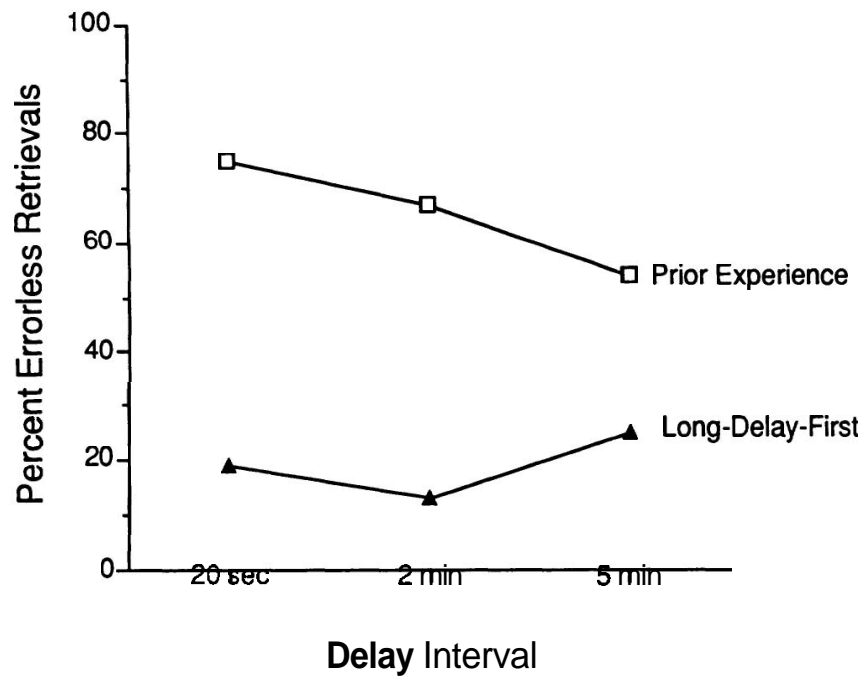

FIG. 3.-Retrieval 1 performance on Day 2 in Study 4. The performance of the Long-delay-first group of Study la is included for comparison.

Loache, 1994). Prior experience with similar tasks can help children in tasks that they would normally fail.

\section{General Discussion}

According to the theoretical account proposed by DeLoache (1990, in press), symbol use requires the achievement of representational insight; that is, one must detect the relation between a symbol and its referent. To do so, one must have a mental representation of the symbol, of the referent, and of the relation between the two. To use a symbol to solve a problem, one must maintain or keep active all three mental representations. If either of the terms of the relation is forgotten or becomes inaccessible for some reason, the representation of the relation itself will necessarily be lost or unavailable.

Our previous studies with scale models have demonstrated that achievement of representational insight is quite difficult for very young children, even with highly iconic symbols. The studies reported here demonstrate that young children have similar difficulty maintaining representational insight.

This conclusion was supported by the finding that children who experienced a long delay on their first trial in the model task performed very poorly on subsequent trials, even those with shorter delays. To clarify this result, compare the experience of the children in the long-delay-first group of Study la to that of the children in the shortdelay-first group. Both groups received detailed instructions about the relation between the highly iconic model and the room, the same instructions as in previous research in which children have performed very well. Thus, we can assume that the children in both groups initially had some, presumably equal, level of representational insight; that is, they had an equivalent awareness of the model-room relation.

All the children then observed the initial hiding event and formed a mental representation of the hiding location of the toy in the model. Because this memory representation was based on direct experience, it was very robust and resistant to decay. In all experiments, the children had little difficulty remembering the location of the toy in the model, regardless of delay or delay order.

After the initial hiding event, the experience of the different groups diverged. Consider the short-delay-first group: Because there was only a short delay, these children began their initial Retrieval 1 attempt with a strong representation of the model-room relation and insight into the relevance of the model to the task. There were two important consequences. First, they successfully retrieved the toy (88\% on trial 1), which reinforced their representation of the modelroom relation. Second, because of their strong representation of the model-room relation and their success in retrieving the toy 


\section{Jttal, Schreiber, and DeLoache 1887}

on the first trial, they construed the task as one in which they should use their experience with the model to guide their action in the room. This appropriate interpretation of the task led them to keep the model-room relation active, even through a longer delay.

A very different fate befell the longdelay-first group. The initial long delay caused their representation of the model and hence of the model-room relation to weaken or become less accessible. We believe that the children were no longer thinking of the model after the initial long delay. The verbal reminder that preceded Retrieval 1 on every trial (reminding the child that the two toys were hidden in the "same places" in their rooms) was insufficient to reinstate their representation of the model. Most of them thus conducted their initial search for the toy in the room with no knowledge of the correct location, so they guessed (38\% correct on trial 1). Having done so, they then construed the task as a guessing game, that is, they adopted their everyday approach to hiding games of searching randomly or simply repeating previously successful searches.

This account clarifies how we were able to ameliorate or prevent the effects of an initial long delay on children's performance. In Studies 2 and $\mathbf{3}$, the children performed much better in the long-delay-first condition if they returned to the model after the initial long delay but before searching in the room. Seeing the model again apparently reminded them of its relevance and hence reinstated the model-room relation for most of the children in Study 2 and for half of the children in Study 3. This visual reminder was thus more effective than our standard verbal reminder ("Remember, Big Snoopy is hiding in the same place as Little Snoopy"). A possible explanation for this difference is that the verbal reminder may not lead children to retrieve their memory of the model (i.e., Little Snoopy's room). We assume that, during the long delay, during which children played with toys or puzzles that were unrelated to the model, the memory of the model faded. A stronger, more salient cue was then required to access it. The sight of the model, which the child readily recognized, would effectively reactivate the child's representation of the model, the hiding event in the model, and the relevance of the model-room relation.

Similar effects of reminders on very young children's performance have been found in research on children's problem solving in different domains. For example, Bullock and Gelman (1977) found that 2year-old children who had learned to choose the array containing the larger (or smaller) number of objects failed to transfer the solution to new array sizes. Children who successfully chose two-object arrays over oneobject arrays failed to select systematically the larger of three- and four-object arrays. However, if the original arrays remained visible, the children were more successful with the new ones. Bullock and Gelman suggested that seeing the original arrays reminded the children that their solution to the previous problems was relevant to the current ones. In our task, seeing the model reminded children of its relevance to searching in the room.

In Study 4, we took a different approach. Instead of reminding children of the relation between the model and the room, we gave them an experience that was designed to strengthen their representation in the first place and hence made it more readily accessible. Children who performed successfully in the standard model task with no delays on Day 1 were also successful on Day 2 in the long-delay-first condition. In the standard, no-delay task on Day 1, achieving representational insight was relatively easy, and the children realized their knowledge of the location of the toy in the model was relevant to searching in the room. Successful performance presumably reinforced their representation of the model-room relation and their correct interpretation of the task. When subjected to a long delay on the first trial on the second day, their firm representation of the model-room relation and their appreciation of the relevance of the model to the room helped these children to tolerate the lengthy delay.

The studies reported here make two contributions to our understanding of representational insight. First, they reveal that becoming aware of a symbol-referent relation is not the final step in exploiting that relation; representational insight is relatively fragile in young children and may not guide performance when there is an impediment, such as a lengthy delay, to keeping both terms of a symbolic relation in mind. We would expect that the degree of fragility of representational insight would be in part a function of the same variables that influence its achievement in the first place. Thus, in the model task, our theoretical account (DeLoache, 1990, in press) would predict that children could more easily keep the model 


\section{Child Development}

and its relevance in mind with higher levels of iconicity, more explicit instructions regarding its relevance, and increased experience with similar symbols. Study 4 demonstrated the last of these; further research is required to test the prediction regarding the first two.

Second, with respect to children's use of scale models per se, the current results indicate that representational insight has to do with children's insight into the overall model-room relation. It has been suggested (e.g., Blades \& Spencer, 1994; Lillard, 1993; Perner, 1991) that children who perform successfully in the model task could do so simply by mapping between the individual objects in the two spaces. That is, they might not recognize any higher level relation between the model and the room but simply notice that the miniature chair in the model corresponds to the larger chair in the room, that the small basket matches the large basket, and so on.

It is plausible that children could succeed in this task purely on the basis of such a set of individual object correspondences. However, our results suggest that success is most likely supported by a higher level representation. The pattern of group differences that we obtained in Study 1 is difficult to explain solely on the basis of correspondences between individual objects. Children might fail with one object pair on the first long delay trial, but there is no reason that the initial failure should influence their performance with subsequent pairs and shorter delays. If they were responding on the basis of object correspondences, trials should be relatively independent of one another. The global influence of the first trial in Study 1 is not easily explained in terms of individual object-to-object correspondences.

The fragility of children's use of a model has parallels to young children's use of other symbols. For example, Liben and Downs $(1989,1992)$ have shown that preschool and young elementary school children who have some insight into the general nature of maps may sometimes lose track of a map's representational nature when interpreting it. They describe one child who correctly identified a body of water on a map of Chicago as Lake Michigan but then identified the compass that represents north as a lifeguard's chair. Another child correctly interpreted a shape on a map as a baseball diamond, but then said the shape next to it was cheese. The children's insight into the mapenvironment relation guided their initial response, but they failed to keep this relation uppermost in mind. Instead, they resorted to sheer physical appearance to make judgments about unknown features of the map. They were no longer thinking about the relation between the map and what it represented in the world.

The maintenance of representational insight may prove to be problematic in other domains as well. For example, in many preschool and elementary school classrooms, teachers use a variety of manipulative aids such as blocks and rods that are intended to help children learn abstract mathematical concepts such as addition and subtraction. Although there is great enthusiasm about the use of such aids, there are some reports that they are not always helpful (Ball, 1992; Goswami, 1992; Hughes, 1986). We would expect that children who have some initial realization that a set of blocks (or another manipulative) is meant to represent numerosity may sometimes lose sight of the symbolic nature of the objects as they manipulate them. Thus, a child might start out correctly using the blocks as symbols but then degenerate into playing with them, thus negating any educational impact (DeLoache, Uttal, \& Pierroutsakos, in press).

As our results and these examples demonstrate, the development of symbolization is neither a straightforward nor a simple process. Even for highly iconic symbols, children must learn that the symbol is a representation of something else and that the relation between the symbol and the referent can be useful for solving problems. Insight into the symbol-referent relation is not, however, sufficient to guarantee consistent or accurate use of the relation. Thus, as children gain knowledge of new symbols, we should expect to see inconsistent and fragile application of this knowledge, followed eventually by skilled and flexible use.

\section{References}

Ball, D. L. (1992). Magical hopes: Manipulatives and the reform of math education. American Educator, 16, 46-47.

Blades, M., \& Spencer, C. (1994). The development of children's ability to use spatial representations. In H. W. Reese (Ed.),Advances in child development and behavior, 25, 157-199.

Bullock, M., \& Gelman, R. (1977). Numerical reasoning in young children: The ordering principle. Child Development, 48, 427-434. 
Crisafi, M. A., \& Brown, A. L. (1986). Analogical transfer of a very young children: Combining two separately learned solutions to reach a goal. Child Deoelopment, 57, 953-968.

Daehler, M., Bukatko, D., Benson, K., \& Myers, N. (1976). The effects of size and color cues on the delayed response of very young children. Bulletin of the Psychonomic Society, 7 , 65-68.

DeLoache, J. S. (1986). Memory in very young children: Exploitation of cues to the location of a hidden object. Cognitiue Deoelopment, 1, 123-137.

DeLoache, J. S. (1987). Rapid change in the symbolic functioning of very young children. Science, 238, 1556-1557.

DeLoache, J. S. (1989). Young children's understanding of the correspondence between a scale model and a larger space. Cognitioe Deuelopment, 4, 121-139.

DeLoache, J. S. (1990). Young children's understanding of models. In R. Fivush \& J. A. Hudson (Eds.), Knowing and remembering in young children (pp. 94-126). Cambridge: Cambridge University Press.

DeLoache, J. S. (1991). Symbolic functioning in very young children: Understanding of pictures and models. Child Deuelopment, 62, 737-752.

DeLoache, J. S. (in press). Early symbolic reasoning. In D. Medin (Ed.), The psychology of learning and motiuation (Vol. 32). New York: Academic Press.

DeLoache, J. S., \& Brown, A. L. (1983). Very young children's memory for the location of objects in a large-scale environment. Child Deuelopment, 54, 888-897.

DeLoache, J. S., Kolstad, V., \& Anderson, K. (1991). Physical similarity and young children's understanding of scale models. Child Deuelopment, 62, 111-126.

DeLoache, J. S., \& Marzolf, D. (1992). When a picture is not worth a thousand words: Understanding the symbolic function of pictures and models. Cognitioe Deoelopment, 7, 317329.

DeLoache, J. S., Uttal, D. H., \& Pierroutsakos, S. L. (in press). The development of early symbolization: Educational implications. Learning and Instruction: The Journal of the European Association on Learning and Instruction.

Ebbinghaus, H. (1913). Memory: A contribution to experimental psychology (H. A. Rutger \& C. E. Bussenius, Trans.). New York: Teachers
College, Columbia University. (Original work published in 1885)

Gentner, D., \& Rattermann, M. J. (1991). Language and the career of similarity. In S. A. Gelman \& J. P. Byrnes (Eds.), Perspectiues on thought and language: Interrelations in deuelopment (pp. 225-277). London: Cambridge University Press.

Gick, M. L., \& Holyoak, K. J. (1983). Schema induction and analogical transfer. Cognitioe Psychology, 15, 1-38.

Goswami, U. (1992).Analogical reasoning in children. East Sussex, England: Erlbaum.

Horn, H., \& Myers, N. (1978).Memory for location and picture cues at ages two and three. Child Deoelopment, 49,845-856.

Holyoak, K. J., Junn, E. N., \& Billman, D. O. (1984). Development of analogical problemsolving skill. Child Deuelopment, 55, 20422055.

Hughes, M. (1986). Children and number: Difficulties in learning mathematics. Oxford: Blackwell.

Liben, L. S., \& Downs, R. M. (1992).Developing an understanding of graphic representations in children and adults: The case of GEOgraphics. Cognitive Deuelopment, 7, 331349.

Liben, L. S., \& Downs, R. M. (1989). Understanding maps as symbols: The development of map concepts in children. In H. W. Reese (Ed.), Adoances in child deoelopment and behaoior (Vol.22, pp. 146-202). San Diego: Academic Press.

Lillard, A. S. (1993). Pretend play skills and the child's theory of mind. Child Deuelopment, 64, 348-371.

Loughlin, K. A., \& Daehler, M. A. (1973). The effects of distraction and added perceptual cues on the delayed reaction of very young children. Child Deoelopment, 44,384-388.

Marzolf, D., \& DeLoache, J. S. (1994). Transfer in young children's understanding of spatial representations. Child Deuelopment, 65, $1-15$.

Perlmutter, M., Hazen, N., Mitchell, D. B., Grady, J. C., Cavanaugh, J. C., \& Flook, J. P. (1981). Pictures cues and exhaustive search facilitate very young children's memory for location. Developmental Psychology, 17, 109-110.

Perner, J. (1991). Understanding the representational mind. Cambridge, MA: MIT Press.

Wynn, K. (1992). Children's acquisition of the number words and counting system. Cognitive Psychology, 24, 220-251. 